\author{
Daniele Di SANTO* - Alessandro B. BisCACCIANTI ${ }^{* *}$
}

\title{
Coleotteri saproxilici in Direttiva Habitat del Parco Nazionale del Gran Sasso e Monti della Laga (Appennino centrale) \\ (Coleoptera Rhysodidae, Lucanidae, Cetoniidae, Cerambycidae)
}

\begin{abstract}
Riassunto: In questa nota si presenta un aggiornamento dei dati sui coleotteri saproxilici inclusi in Direttiva Habitat presenti nel territorio del Parco Nazionale del Gran Sasso e Monti della Laga. Sono state censite cinque specie, Rhysodes sulcatus, Lucanus cervus, Osmoderma eremita, Cerambyx cerdo e Rosalia alpina, per ognuna delle quali, oltre a riportarne i dati di cattura e bibliografici a noi noti, se ne discute brevemente il significato ecologico e conservazionistico. Lucanus cervus è qui citato per la prima volta dell'Abruzzo e, assieme a Rhysodes sulcatus e C. cerdo, anche per il territorio dell'area protetta. Il rinvenimento di $R$. sulcatus risulta particolarmente significativo sia per la rarità della specie, sia perché il reperto qui riportato ne conferma la presenza nell'Italia peninsulare.
\end{abstract}

\begin{abstract}
Saproxylic beetles of Habitats Directive of the Gran Sasso e Monti della Laga National Park (Central Apennine). A review of data on saproxylic beetles listed in the EU Habitats Directive occurring in the Gran Sasso e Monti della Laga National Park is presented. Five species, Rhysodes sulcatus, Lucanus cervus, Osmoderma eremita, Cerambyx cerdo, and Rosalia alpina have been recorded; known bibliographic references and collecting data are provided, together with a brief discussion on their ecological and conservation significance. Lucanus cervus is here quoted for the first time for the Abruzzo region and, together with Rhysodes sulcatus and C. cerdo, also for the National Park. The record of $R$. sulcatus is particularly remarkable for its rarity and confirms the occurrence of this endangered species in peninsular Italy.
\end{abstract}

Key words: Saproxylic Coleoptera, Habitats Directive, Italy, Faunistics, Ecology.

\section{INTRODUZIONE}

Il Parco Nazionale del Gran Sasso e Monti della Laga (Fig. 1) si estende per circa 149.000 ettari nell'Appennino centrale, a cavallo delle regioni Marche, Lazio e Abruzzo. E' il terzo Parco Nazionale italiano per estensione, dopo il Parco Nazionale del Pollino (Appennino calabro-lucano) e il Parco Nazionale del Cilento, Vallo di Diano e Alburni (Appennino campano). Il territorio dell'area protetta è caratterizzato dalla presenza di tre gruppi montuosi principali: la catena del Gran Sasso nel settore centro-meridionale, costituita da rocce calcaree e dolomitiche, il gruppo dei Monti Gemelli nel settore nord-orientale, anch'esso di natura calcarea, e il massiccio dei Monti della Laga nel settore centro-settentrionale, costituito da rocce marnoso-arenacee. La massima elevazione è rappresentata dal Corno Grande (2912 m s.1.m.), che è anche la vetta più alta della catena appenninica.

Gli ambienti forestali occupano oltre il 60\% (circa 87.000 ettari) della superficie totale dell'area protetta e, analogamente ad altri gruppi montuosi
dell'Italia centrale, presentano fisionomie vegetazionali piuttosto eterogenee (cfr. Tammaro, 1998; Collalti et al., 2009). La tipologia forestale più diffusa è rappresentata dalle faggete, talvolta caratterizzate dalla presenza di abete bianco, tasso e agrifoglio; esse si estendono prevalentemente sui versanti settentrionale e orientale del Gran Sasso e sui Monti della Laga. Nel piano collinare-submontano sono frequenti cerrete, boschi misti termofili a dominanza di roverella e castagneti, sebbene tutti alquanto frammentati e degradati. Sono inoltre presenti formazioni più o meno estese di sclerofille mediterranee a dominanza di leccio, che vegetano soprattutto nei settori orientale e meridionale del Gran Sasso. Oltre alle tipologie sopraccitate, nel territorio del Parco si riscontrano interessanti nuclei relitti di betulla, carpino bianco e boschi di forra a dominanza di frassino maggiore, con tiglio e olmo montano (Hruska, 1988; Tondi, 1996; Tammaro, 1998; Collalti et al., 2009).

Nonostante l'intenso, secolare sfruttamento di tali boschi, nell'area del Parco permangono lembi di

\footnotetext{
*Daniele Di Santo, Ente Parco Nazionale del Gran Sasso e Monti della Laga, Via del Convento 1, 67010 Assergi (AQ), Italia. E-mail: danieledisanto@gransassolagapark.it.

${ }^{* *}$ Alessandro B. Biscaccianti, Via Simeto 12, 00198 Roma, Italia. E-mail: a.biscaccianti@libero.it
} 
foresta ben conservata, spesso con elevato grado di vetustà (Calamini et al., 2011), quali ad esempio le faggete di Fonte Novello (Di Santo et al., 2010) (Fig. 2), Aschiero (Fig. 3), Martese, S. Gerbone e il frassineto di Valle Vaccaro.

Scopo di questo contributo è fornire un quadro aggiornato sulla presenza e distribuzione delle specie di coleotteri saproxilici incluse in "Direttiva Habitat" (Consiglio CE, 1992; Consiglio UE, 2006) nel territorio del Parco Nazionale del Gran Sasso e Monti della Laga.

\section{MATERIALI E METODI}

I dati qui riportati derivano in gran parte dalle ricerche effettuate dagli autori nell'ambito del progetto "Identificazione, mappatura, caratterizzazione strutturale e pianificazione dei boschi vetusti del Parco Nazionale del Gran Sasso e Monti della Laga", svolto grazie a un accordo di collaborazione tra il suddetto Ente Parco e l'Università della Tuscia, Dipartimento per l'Innovazione nei sistemi Biologici, Agroalimentari e Forestali (Viterbo). Altri dati inediti derivano da ricerche occasionali da noi effettuate in varie località del Parco, o da segnalazioni comunicateci da colleghi o semplici appassionati.

Per ciascuna specie si riportano i dati completi di osservazione o di cattura, le segnalazioni bibliogra-

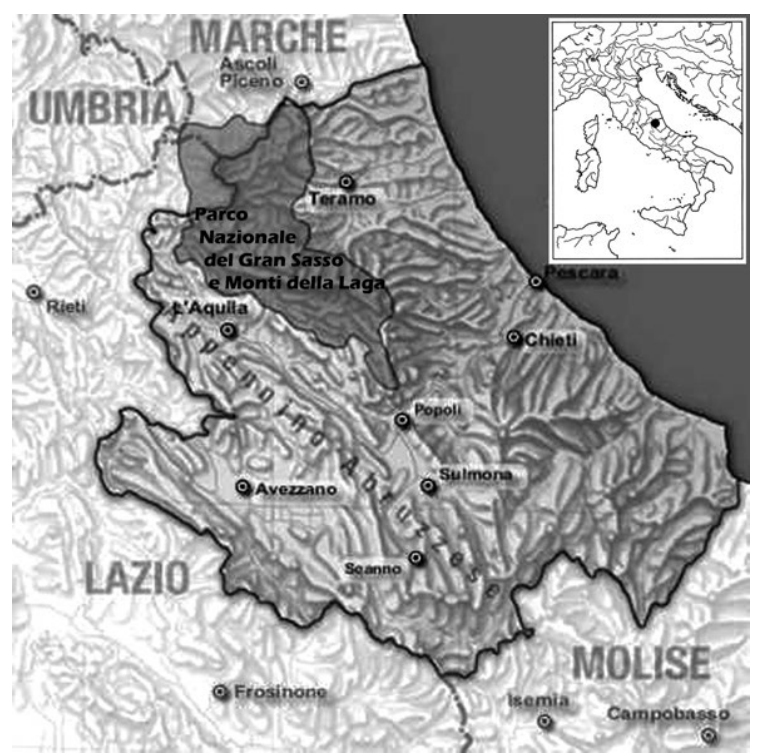

Fig. 1. Inquadramento territoriale dell'area di studio. fiche, se disponibili, i principali riferimenti normativi di tutela comunitaria e una nota di commento; eventuali interpolazioni sono tra parentesi quadre. Nel testo sono state adottate le abbreviazioni riportate più avanti. Salvo diversa indicazione, il materiale raccolto è conservato in collezione Biscaccianti.

La nomenclatura zoologica segue, con alcune eccezioni, quella adottata nei volumi del Catalogue of Palaearctic Coleoptera (Löbl \& Smetana, 2003, 2004, 2006, 2007, 2008, 2010), mentre quella botanica fa riferimento a Conti et al. (2005) per le piante vascolari, e al database online Species Fungorum (http://www. speciesfungorum.org) per i funghi e i mixomiceti.

AbBreviazioni. $\mathrm{AB}=$ vidit/legit A.B. Biscaccianti; $\mathrm{ALi}=$ collezione $\mathrm{A}$. Liberto, Roma; $\mathrm{AP}=$ Marche, provincia di Ascoli Piceno; $A Q=A b r u z z o$, provincia de L'Aquila; BD=viderunt/legerunt A.B. Biscaccianti e D.

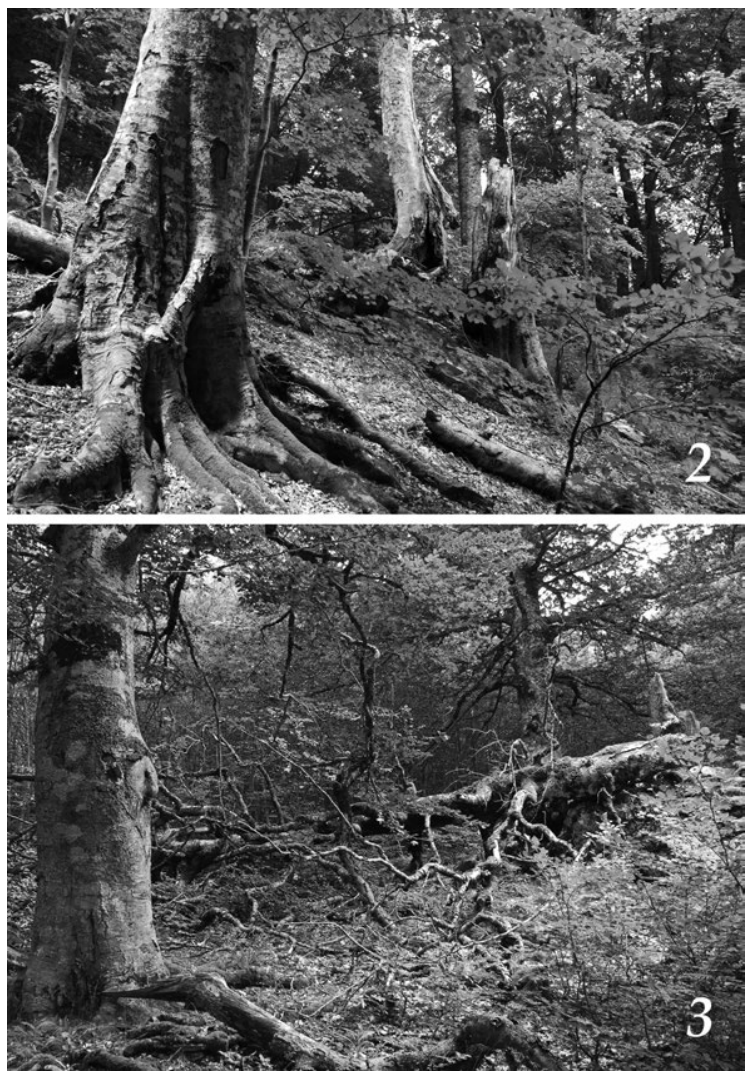

Figg. 2-3. Faggete vetuste del Gran Sasso: 2. Bosco di Fonte Novello; 3. Bosco Aschiero. (foto D. Di Santo). 
Di Santo; DD=vidit/legit D. Di Santo; ex-exx=esemplare-i; loc. $=$ località/stazione; $\mathrm{MCZR}=$ Museo Civico di Zoologia, Roma; ME=vidit M. Ettorre; RI=Lazio, provincia di Rieti; $\mathrm{sl}=$ senza legit; $\mathrm{TE}=\mathrm{Abruzzo}$, provincia di Teramo.

\section{RISULTATI}

Rhysodes sulcatus (Fabricius, 1787) (Rhysodidae) Normativa COMUNITARIA DI TUTELA. Direttiva 2006/ 105/CE [adeguamento della Direttiva 92/43/CEE], Allegato II (Consiglio UE, 2006).

Materiale esaminato. 10 , Gran Sasso, Pietracamela (TE), M. Corvo NE, Valle Venacquaro loc. Piana Grande, m 1220, 10-20.V.2012, ex larva (prelievo 11396 del 6.IX.2011), BD.

Specie ovunque assai rara e sporadica, diffusa in gran parte dell'Europa, raggiunge a Est gli Urali meridionali, la regione caucasica e transcaucasica e l'Asia minore (Brustel \& Gouix, 2011). In Italia è nota di Lombardia, Emilia-Romagna, Toscana, Lazio, Abruzzo, Campania e Basilicata (Porta, 1923; Luigioni, 1929; Papini, 1962; Cecchi \& Bartolozzi, 1997). Dell'Abruzzo era già nota per un ritrovamento nel Parco Nazionale d'Abruzzo, Lazio e Molise (Papini, 1962: "Vallone Pesco di Lordo, m 1500") ed è quindi interessante la sua scoperta anche sul Gran Sasso.

Rhysodes sulcatus è ecologicamente legato alla presenza abbondante di legno morto di grosso calibro colonizzato da funghi saprofiti e mixomiceti. E' infatti un elemento silvicolo stenotopo e stenoecio, esclusivo di foreste primarie o con elevato grado di vetustà (cfr. Speight, 1989; Franklin \& Spies, 1991; Keddy \& Drummond, 1996; Gilg, 2004). Larve e adulti di $R$. sulcatus vivono nel legno deiscente a terra di latifoglie e conifere (Burakowski, 1975), ove si nutrono presumibilmente degli stadi ameboidi di mixomiceti (Bell, 1998); la biologia di questa specie, e dei Rhysodidae in generale, è tuttavia poco nota (Makarov, 2008).

L'esemplare qui citato è stato ottenuto ex larva da campioni di legno di Fagus sylvatica L. nei quali erano presenti carpofori di Ganoderma applanatum (Pers.) Pat. (Polyporales, Ganodermataceae) e sporofori di Lycogala epidendrum (J.C. Buxb. ex L.) Fr. (Liceida, Tubiferaceae), prelevati da un grosso tronco marcescente al suolo. Dagli stessi campioni sono stati ottenuti anche esemplari di Odontosphindus grandis (Hampe, 1861) (Sphindidae), altra significativa specie legata a mixomiceti, recentemente segnalata per l'Italia (Audisio et al., 2008), Anisotoma humeralis (Fa- bricius, 1792) (Leiodidae), specie poco frequente legata al legno marcescente di grossa mole, e altre specie di coleotteri legate a funghi saprofiti.

Rhysodes sulcatus è stato recentemente incluso tra le specie tutelate dalla Direttiva Habitat (vedi sopra) in quanto seriamente a rischio di estinzione in molti stati europei (cfr. Nieto \& Alexander, 2010) a seguito della progressiva scomparsa e frammentazione delle foreste vergini e semivergini.

Lucanus (Lucanus) cervus cervus (Linnaeus, 1758) (Lucanidae)

Normativa Comunitaria Di tutela. Convenzione di Berna, Allegato III (Council of Europe, 1979); Direttiva 92/43/CEE, Allegato II (Consiglio CE, 1992); Direttiva 2006/105/CE [adeguamento della Direttiva 92/43/CEE], Allegato II (Consiglio UE, 2006).

Materiale esaminato. $1 \delta^{\lambda}, 1$, Monti della Laga, Valle Castellana (TE), Capolaterra, m 650, 17.VII.1999, AB.

Specie suddivisa in varie sottospecie (Bartolozzi \& Sprecher-Uebersax, 2006), alcune delle quali probabilmente da considerarsi specie valide, anche se il loro effettivo status necessita di ulteriori studi a livello molecolare (Cox et al., 2013). La sottospecie nominale è ampiamente diffusa in Europa, Kazakhstan, Caucaso e Asia minore (Bartolozzi \& Sprecher-Uebersax, 2006). In Italia è nota di tutte le regioni settentrionali e centrali fino al Lazio, Umbria, Marche (Bartolozzi \& Maggini, 2005) e Abruzzo (qui segnalata per la prima volta); le citazioni per le regioni meridionali necessitano di conferma e quella di Sicilia è quasi sicuramente dovuta a un errore di cartellinatura di un vecchio esemplare museale (Bartolozzi, com. pers.). Nell'Italia centrale e centro-settentrionale $L$. cervus è in contatto e talora sovrapposto con l'affine, semivicariante, L. tetraodon Thunberg, 1806, che lo sostituisce nelle regioni meridionali e in Sicilia. La citazione per la Sardegna di L. cervus (cfr. Franciscolo, 1997) e quella per la Liguria di L. tetraodon (cfr. Bartolozzi \& Maggini, 2005) sono da ritenersi dubbie (Bartolozzi, com. pers.), mentre di recente popolazioni di quest'ultima specie sono state invece trovate in Lombardia (Zilioli \& Pittino, 2004) ed Emilia Romagna (Fabbri, 2010); L. cervus non era ancora stato segnalato per il Parco Nazionale del Gran Sasso e Monti della Laga, tuttavia è nota una vecchia cattura effettuata ad Ascoli Piceno (Bartolozzi \& Maggini, 2005), località marchigiana non lontana dai confini settentrionali del Parco stesso. 
Lucanus cervus è una specie xilosaprobia legata ai boschi di latifoglie del piano basale e collinaresubmontano, prevalentemente querceti e castagneti, ma presente talvolta anche in boschi misti, faggete $\mathrm{e}$ foreste riparie. Sebbene sia più frequente in comprensori forestali maturi e strutturalmente evoluti, si rinviene non di rado anche in ambienti degradati, quali boscaglie di sostituzione, boschi cedui, singoli alberi o filari inseriti in matrici agricole, oltreché in parchi urbani e suburbani. Le larve sono notevolmente polifaghe, scavano gallerie trofiche in profondità nel legno deiscente di tronchi morti, parti morte di vecchi alberi e ceppaie, per un periodo compreso fra tre e otto anni, per poi impuparsi a fine estate nel terreno, protette da un caratteristico bozzolo. Gli adulti, che svernano nel suddetto bozzolo, presentano una fenologia prevalentemente estiva (Giugno-Settembre) e costumi crepuscolari (Franciscolo, 1997).

Secondo Harvey et al. (2011), L. cervus, pur non essendo una specie a rischio di estinzione, è comunque in regressione in alcune parti d'Europa a causa dell'eccessiva frammentazione del suo habitat e della sistematica rimozione del legno morto dai comprensori forestali.

Osmoderma eremita (Scopoli, 1763) (Cetoniidae) Normativa COMUnitaria Di TUTEla. Convenzione di Berna, Allegato II (Council of Europe, 1979); Direttiva 92/43/CEE, Allegati II (specie prioritaria) e IV (Consiglio CE, 1992); Direttiva 2006/105/CE [adeguamento della Direttiva 92/43/CEE], Allegati II (specie prioritaria) e IV (Consiglio UE, 2006).

Segnalazioni bibliografiche. Gran Sasso: Abruzzo (TE), Vallone Venacquaro (Carpaneto et al., 2005). Materiale esaminato. $1 \hat{\jmath}$, Gran Sasso, Pietracamela (TE), M. Corvo NE, Valle Venacquaro loc. Fonte Novello, m 1220, 6.IX.2011, BD.

La sistematica del complesso di specie, o semispecie, o "evolutionarily significant unit" (sensu Moritz, 1994), delle Osmoderma Le Peletier \& Audinet-Serville, 1828 del gruppo eremita è controversa (cfr. Sparacio, 1994, 2000; Tauzin, 1994; Krell, 1997; Audisio et al., 2003, 2007, 2009; Brustel, 2004) e lo status dei taxa a esso riferibili non è ancora del tutto definito $(\mathrm{Au}-$ disio et al., 2007, 2009). Le popolazioni dell'Abruzzo sono da ascrivere a $O$. eremita s.str.; occorre tuttavia precisare che all'epoca dell'emanazione della Direttiva Habitat tutte le popolazioni italiane di Osmoderma si riteneva fossero appartenenti all'unica specie $O$. ere- mita. In seguito, lo studio accurato di materiale delle regioni meridionali e insulari d'Italia ha portato alla separazione di O. cristinae Sparacio, 1994 (Sicilia) e di O. italicum Sparacio, 2000 (Italia meridionale) (cfr. Sparacio, 2000; Audisio et al., 2003, 2007, 2009).

Osmoderma eremita è diffusa in Europa centrooccidentale, a Nord fino alla Penisola Scandinava meridionale, a Est fino all'Austria e alla Germania occidentale (a contatto sovrapposto con $O$. barnabita Motschulsky, 1845), a Ovest fino alla regione pirenaica, parte della Spagna settentrionale e a tutta la Francia, a Sud fino all'Italia centrale (Audisio et al., 2007). In Italia meridionale è vicariata da $O$. italicum (endemita appenninico diffuso dalla Campania all'Aspromonte), mentre in Sicilia è sostituita da $O$. cristinae (endemita siculo) (Audisio et al., 2003, 2007; Carpaneto et al., 2005). Osmoderma eremita è piuttosto diffusa in Abruzzo ed era già nota anche del Parco Nazionale del Gran Sasso e Monti della Laga e di alcune località prossime ai confini occidentali e meridionali dello stesso (Sparacio, 2000; Carpaneto et al., 2005; Biscaccianti, 2006). Il biotopo di Fonte Novello è tuttavia di particolare interesse anche per la presenza sintopica di Protaetia marmorata (Fabricius, 1792) (Cetoniidae), specie rara e molto localizzata in Italia, legata anch'essa alle cavità degli alberi vetusti e valido indicatore del grado di naturalità degli ecosistemi forestali (Oleksa et al., 2013).

L'habitat primario delle specie italiane di Osmoderma è rappresentato dai boschi maturi con presenza di vecchi alberi cavi, dal piano basale a quello montano. Tuttavia, mentre $O$. eremita s.str. è presente localmente anche in ambienti a parziale influenza antropica, purché permangano grandi alberi cariati, O. italicum, e $O$. cristinae, sono assai più localizzate e legate ad ambienti strutturalmente più evoluti (Audisio et al., 2003; Audisio \& Biscaccianti, dati inediti). Le larve sono xilosaprobie e si sviluppano negli accumuli di rosura all'interno delle parti cariate di alberi senescenti di numerose latifoglie, prevalentemente querce, faggi, salici e castagni (Prunier, 1999; Ranius, 2000, 2002b). Lo sviluppo preimmaginale richiede tre anni solari e l'impupamento avviene all'interno di un bozzolo formato da escrementi e rosura compattati. Gli adulti sono attivi in primavera-estate (Giugno-Agosto) nelle ore pomeridiane e sono generalmente poco vagili (Ranius \& Hedin, 2001).

Sotto il profilo conservazionistico, la tutela del peculiare habitat delle specie appartenenti al genere Osmoderma è di primaria importanza sia per le carat- 
teristiche autoecologiche di queste, sia in relazione all'entomofauna ad esse associata, che annovera elementi spesso altamente specializzati e quindi assai vulnerabili e sensibili alle alterazioni ambientali (Ranius \& Nilsson, 1997; Dajoz, 2000; Ranius, 2000, 2001, 2002a; Biscaccianti \& Casalini, 2007).

Cerambyx cerdo cerdo Linnaeus, 1758 (Cerambycidae)

Normativa COMUnitaria Di TUTEla. Convenzione di Berna, Allegato II (Council of Europe, 1979); Direttiva 92/43/CEE, Allegati II e IV (Consiglio CE, 1992); Direttiva 2006/105/CE [adeguamento della Direttiva 92/43/CEE], Allegati II e IV (Consiglio UE, 2006).

Materiale esaminato. 1 9 , 2 exx (resti), Gran Sasso, tra Capestrano e Ofena (AQ), m 400-500, 1113.VIII.2005, AB.

Specie ad ampia distribuzione suddivisa in due sottospecie, C. c. cerdo e C. c. mirbeckii Lucas, 1842. La sottospecie nominale occupa gran parte dell'Europa, Caucaso, Transcaucasia, Asia Minore, Medio Oriente e Nord Africa (Marocco), ma è maggiormente diffusa nell'Europa centro-meridionale e nell'area mediterranea (Bense, 1995; Sama, 2002); la sottospecie mirbeckii è nota di Marocco, Algeria e Tunisia. In Italia C. cerdo è presente in tutte le regioni eccetto la Valle d'Aosta (Sama, 2005), ma sembra più localizzata nelle regioni centro-meridionali, probabilmente per difetto di ricerche. Per l'Abruzzo esisteva unicamente una citazione basata su vecchi reperti (cfr. Sama, 1988, 2005), ma le indagini condotte negli anni da uno di noi (Biscaccianti, 2006 e dati inediti) ne hanno accertato la presenza in numerose stazioni, ivi compresa quella qui citata relativa al Gran Sasso.

Cerambyx cerdo è una specie xilofaga ecologicamente legata a cenosi forestali termofile e xerotermofile (querceti, boschi misti termofili, sugherete, leccete), con presenza di piante senescenti e debilitate. Nonostante sia citata come ospite di numerose essenze arboree, è essenzialmente stenofaga a spese di Quercus spp., di cui attacca grossi rami e tronchi di piante vegete, preferibilmente vecchi alberi debilitati (Sama, 1988, 2002; Bense, 1995; Buse et al., 2008a). E' localmente abbastanza frequente nel piano basale e collinare, ma può talvolta raggiungere il piano montano inferiore. La larva scava una lunga galleria trofica dapprima sotto la corteccia e successivamente in profondità nel legno vivo, ove avviene anche l'impupamento. Lo sviluppo preimmaginale richiede mediamente tre o quattro anni, ma le larve non sopravvivono se l'albero muore o viene abbattuto. L'adulto ha fenologia primaverile-estiva (Giugno-Agosto) e costumi crepuscolari. Nella località citata $C$. cerdo è stato raccolto su Quercus pubescens Willd. ai margini di un incolto assieme a $C$. welensii (Küster, 1845), con il quale spesso convive. Nelle gallerie subcorticali dei due longicorni è stato raccolto Teredus cylindricus (Olivier, 1790) (Bothrideridae), raro saproxilico spesso associato alle gallerie trofiche di Cerambyx spp. (Biscaccianti, dati inediti).

Cerambyx cerdo è ovunque in lenta regressione per la progressiva scomparsa del suo habitat primario, costituito dai boschi radi o a copertura discontinua con presenza di alberi vetusti (Albert et al., 2012). É tuttavia una specie che si adatta facilmente agli ambienti urbani colonizzando con successo le vecchie querce ornamentali delle ville storiche e dei viali cittadini, considerate in genere patrimonio storico-culturale.

Pur non essendo attualmente a rischio di estinzione se non localmente, l'eccessivo sfruttamento dei boschi planiziari e collinari, la loro frammentazione, l'eliminazione di piante senescenti, debilitate e, più in generale, la semplificazione strutturale delle foreste di media e bassa quota, sono le cause principali della progressiva rarefazione di C. cerdo in Italia, dove era assai comune fino a pochi decenni orsono. La tutela di questa specie è direttamente connessa con la necessità di preservare l'habitat cui è legata, anche perché di primaria importanza per numerosi altri organismi più specializzati e vulnerabili. Infatti, come per altre specie di insetti xilofagi, l'attività trofica delle larve contribuisce a incrementare la disponibilità di legno morto su alberi vegeti, favorendo il mantenimento di importanti comunità di artropodi saproxilici (cfr. Jones et al., 1994; Grove, 2002; Travé, 2003; Buse et al., 2008a, 2008b).

Rosalia alpina alpina (Linnaeus, 1758) (Cerambycidae)

NoRmativa COMUnitaria DI TUTELA. Convenzione di Berna, Allegato II (Council of Europe, 1979); Direttiva 92/43/CEE, Allegati II (specie prioritaria) e IV (Consiglio CE, 1992); Direttiva 2006/105/CE [adeguamento della Direttiva 92/43/CEE], Allegati II (specie prioritaria) e IV (Consiglio UE, 2006).

Segnalazioni Bibliografiche. Monti della Laga: Marche (AP), Bosco di San Gerbone, m 1100; Abruzzo (TE), Bosco della Martese loc. Ceppo, $m$ 1300 (Gobbi, 1994). 
Materiale esaminato. $1{ }^{\lambda}$, Monti della Laga, Amatrice (RI), Capricchia loc. Costa Amata, m 1400, VIII.1986, AB; 1ô, 1옹 Monti della Laga, Rocca Santa Maria (TE), Bosco della Martese, Rio Castellano, m 1000, 7.VIII.1992, AB; 1 $^{\lambda}$, Monti della Laga, Rocca Santa Maria (TE), Bosco della Martese loc. Ceppo, m 1300, 15.VII.1994, A. Liberto legit, ALi; 1 ex, Monti della Laga, Crognaleto (TE), M. di Mezzo SE, loc. Folgora-Pianocchie, m 1450, VIII.2008, ME; 1 ex, Monti della Laga, Crognaleto (TE), M. di Mezzo SE, loc. Le Cannavinelle, m 1250, VIII.2008,

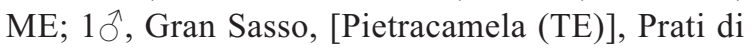
Tivo, VIII.1984, sl; 1엉 ibidem, 27.VII.1997, sl (MCZR); 19, Gran Sasso, Fano Adriano (TE), 5.VIII.1997, sl (MCZR); 1 ex, Gran Sasso, Pietracamela (TE), M. Corvo NE, Valle Venacquaro loc. Pretara (particella forestale 301), m 1180, estate 2006, ME; 2 larve, Gran Sasso, Pietracamela (TE), M. Corvo NE, Valle Venacquaro loc. Fonte Novello, m 1220, 14.VI.2011, BD; 19, ibidem, 6.IX.2011, BD;

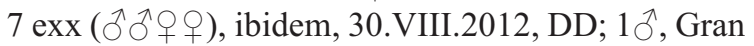
Sasso, Pietracamela (TE), M. Corvo NE, Valle Venacquaro loc. Pagliarone, m 1100, 6.IX.2011, BD; 1 ex, Gran Sasso, Pietracamela (TE), Val Maone, 5.VIII.2010, L. Troiani vidit; $1 \partial^{\lambda}$, Gran Sasso, Pietracamela (TE), Val Maone, sentiero per Passo Portella, 7.VIII.2010, S. Agabio e D. Zanella viderunt; 1 , Gran Sasso, Isola del Gran Sasso (TE), fraz. Pretara, Fosso Ruzzo loc. Cascata della Costa dei Baroni, m 750, 14.IX.2013, C. Artese vidit; $1{ }^{\lambda}$, Gran Sasso, Crognaleto (TE), Prati di Incodara, Rifugio Forestale, m 1420, 13.IX.2013, DD; 1q, Gran Sasso, L'Aquila (AQ), Bosco del Chiarino loc. Colle dei Briganti, $\mathrm{m}$ 1500 ca., 2.VIII.2012, DD.

Specie suddivisa in due sottospecie, $R$. $a$. alpina (Fig. 4) e R. a. syriaca Pic, 1894, il cui valore tassonomico appare tuttavia assai discutibile. La sottospecie nominale è diffusa in gran parte dell'Europa, a Nord fino alla Francia e alla Polonia centro-settentrionali, a Est fino alla Bielorussia, Ucraina, Caucaso e Transcaucasia e a Sud fino alla Spagna settentrionale, Italia meridionale (Sicilia), Grecia (Peloponneso) e Turchia settentrionale; la sottospecie syriaca è nota della Turchia sud-orientale (cfr. Sama, 2002).

Rosalia alpina è nota di tutte le regioni italiane eccetto la Sardegna, ma la sua presenza in Puglia (Costa, 1854: "Gargano") necessita di conferma (Biscaccianti, 2004). In ambito appenninico la specie è ancora piuttosto diffusa e relativamente frequente, mentre è più localizzata nell'arco alpino e in Sicilia (Biscaccianti, 2004 e dati inediti; Bosso et al., 2013). Per l'Abruzzo la specie era nota unicamente di alcune località dei Monti Marsicani, dei Monti della Meta (Sama, 1988, 2005) e dei Monti della Laga (Gobbi, 1994); è invece molto più diffusa sia nella regione (Biscaccianti, 2006 e dati inediti), sia nel Parco Nazionale del Gran Sasso e Monti della Laga (dati qui riportati).

Rosalia alpina è ecologicamente legata a cenosi di latifoglie mesofile, il cui habitat primario è rappresentato dalle faggete mature con presenza di grandi alberi senescenti e morti, ceppi, legno morto a terra di grossa mole (cfr. Demelt, 1966; Contarini, 2003; Biscaccianti, 2004; Russo et al., 2011; Castro et al., 2012). Trattandosi di una specie eliofila, necessita inoltre di aree aperte all'interno della copertura boschiva, o comunque di un mosaico forestale discontinuo in cui la risorsa trofica sia disponibile in ambito ecotonale o nelle radure. Sebbene si sviluppi prevalentemente a spese di Fagus spp., è in grado di colonizzare occasionalmente anche altre latifoglie (cfr. Bense, 1995), in particolare Fraxinus spp. Le larve sono xilofaghe e si sviluppano dapprima sotto la corteccia e successivamente in profondità nel legno di grossi alberi morti o nelle parti morte di piante senescenti. Lo sviluppo larvale richiede mediamente due

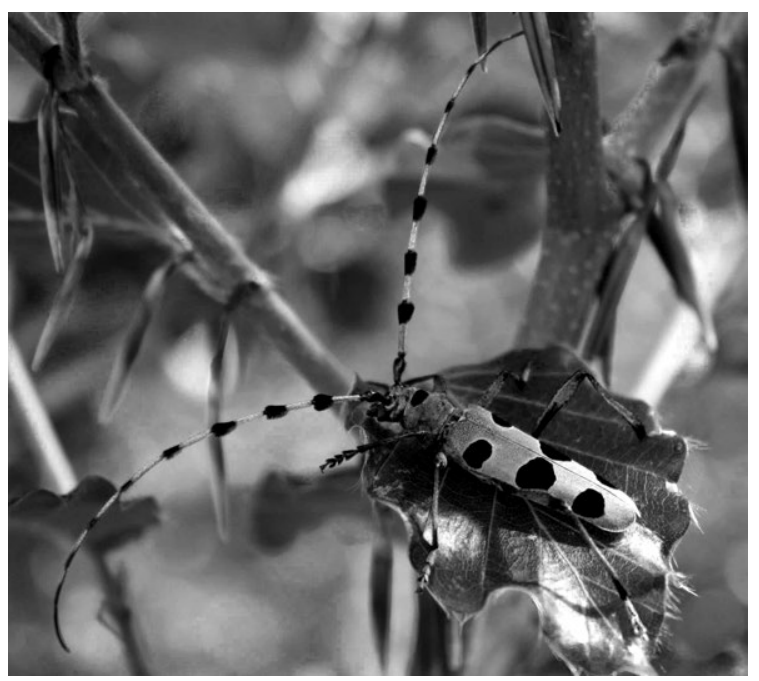

Fig. 4. Maschio di Rosalia alpina della Val Maone (foto S. Agabio e D. Zanella). 
o tre anni solari, l'impupamento avviene all'interno del legno tra la fine della primavera e l'inizio dell'estate, cui segue lo sfarfallamento degli adulti, generalmente tra la terza decade di Giugno e la seconda decade di Agosto; questi sono attivi fino alla prima decade di Settembre, sono diurni ed eliofili e frequentano i tronchi e i grossi rami esposti dei faggi morti o senescenti, ma anche le cataste di legna.

Come si è detto, $R$. alpina è piuttosto diffusa in tutto il territorio del Parco Nazionale del Gran Sasso e Monti della Laga. Nei boschi meglio conservati della Valle del Venacquaro la specie è insediata con popolazioni consistenti ed è quasi ovunque associata a Morimus asper (Sulzer, 1776) (Cerambycidae). Il biotopo presenta un'elevata idoneità ambientale per $R$. alpina, confermata anche dalla presenza sintopica di Acanthocinus xanthoneurus (Mulsant \& Rey, 1852) (Cerambycidae), raro endemita appenninico e valido indicatore del grado di naturalità delle faggete appenniniche (cfr. Biscaccianti, 2004; Blasi et al., 2010).

Rosalia alpina è tuttavia in regressione in gran parte dell'Italia a causa della progressiva scomparsa e frammentazione del suo habitat naturale: la semplificazione strutturale delle foreste mesofile, l'eliminazione dai boschi gestiti degli alberi senescenti, debilitati o morti e l'eccessiva frammentazione dei nuclei di foresta vetusta, rappresentano le principali cause di rarefazione e, in molti casi, di estinzione di questo vistoso longicorne. Il carattere ormai "insulare" delle faggete idonee allo sviluppo di $R$. alpina, infatti, rende spesso impossibile lo scambio tra le diverse popolazioni, che risultano pertanto isolate e maggiormente vulnerabili.

\section{CONCLUSIONI}

La recente versione consolidata della Direttiva Habitat (Consiglio UE, 2006) elenca 21 specie di coleotteri saproxilici (sensu Speight, 1989), di cui almeno 9 presenti anche in Italia: Rhysodes sulcatus (Rhysodidae), Lucanus cervus (Lucanidae), Osmoderma eremita (s.1.) (Cetoniidae), Buprestis splendens Fabricius, 1775 (Buprestidae), Stephanopachys substriatus (Paykull, 1800) (Bostrichidae), Cucujus cinnaberinus (Scopoli, 1763) (Cucujidae), Cerambyx cerdo, Morimus funereus Mulsant, 1862 (sensu Reitter, 1894) e Rosalia alpina (Cerambycidae). A queste occorre forse aggiungere Bolbelasmus unicornis (Schrank, 1789) (Geotrupidae), specie generalmente legata ad ambienti forestali ma non strettamente saproxilica, di cui però si conosce ancora poco della sua biologia ed ecologia (Koch, 1989; Nadai, 2006; Jurc et al., 2008; Paill, 2008). E' invece alquanto dubbia la presenza in Italia (Alto Adige) di Stephanopachys linearis (Kugelann, 1792) (Bostrichidae) (cfr. Nardi, 2005): questa specie è citata erroneamente da Lesne (1901, sub S. elongatus Paykull, 1800) e da Horion (1961) sulla base di presunti dati di Gredler (1866), il quale invece non la menziona affatto; la sua presenza in Alto Adige non è infatti confermata da Peez \& Kahlen (1977).

Tralasciando le problematiche di natura tassonomica e normativa che riguardano alcuni taxa (Osmoderma spp., Morimus spp.), argomento che esula dallo scopo di questo contributo, va detto che delle nove specie presenti in Italia, solamente quattro (L. cervus, O. eremita, C. cerdo, R. alpina) sono diffuse in tutto o gran parte del territorio italiano (Ruffo \& Stoch, 2005; Biscaccianti, dati inediti), e sono sufficientemente conosciute sotto il profilo biologico, ecologico e geonemico. Le restanti specie sono invece estremamente rare e localizzate (Rhysodes sulcatus, Cucujus cinnaberinus), presenti in aree molto ristrette (Buprestis splendens) o marginali del territorio italiano (S. substriatus, M. funereus, Bolbelasmus unicornis) e, di conseguenza, le conoscenze sulla loro biologia ed ecologia sono ancora decisamente lacunose. A questo si aggiunge la carenza di studi specifici sugli invertebrati di Direttiva Habitat in generale, spesso trascurati anche nell'ambito delle indagini per la redazione dei piani di gestione dei siti Natura 2000.

Rhysodes sulcatus e Rosalia alpina sono entrambe specie caratteristiche delle foreste mesofile del piano submontano e montano, la prima associata ai grossi tronchi marcescenti al suolo colonizzati da funghi saprofiti e mixomiceti, la seconda legata prevalentemente al legno asciutto dei vecchi faggi morti in foresta o nelle radure. Nei biotopi meglio conservati del Parco, l'habitat di Rhysodes sulcatus è spesso colonizzato da interessanti coleotterocenosi saproxiliche, tra cui spiccano specie rare e stenotope come l'endemita appenninico Derodontus raffrayi Grouvelle, 1917 (Derodontidae), Odontosphindus grandis (Sphindidae), Denticollis linearis (Linnaeus, 1858) (Elateridae), $\mathrm{Me}$ landrya caraboides (Linnaeus, 1760) (Melandryidae) e altre, mentre nei grossi faggi morti più esposti, colonizzati da Rosalia alpina, si insediano elementi altrettanto significativi come Bothrideres bipunctatus (Gmelin, 1790) (Bothrideridae), Acanthocinus xanthoneurus 
(Cerambycidae) e Pediacus dermestoides (Fabricius, 1792) (Cucujidae), solo per citarne alcuni.

Lucanus cervus e Cerambyx cerdo sono invece specie di bassa quota, legate prevalentemente ai boschi misti termofili, alle boscaglie di querce e talvolta ai vecchi alberi che vegetano nelle matrici agricole. Lucanus cervus è qui ai margini meridionali dell'areale italiano, a contatto sovrapposto con l'affine $L$. tetraodon, specie a gravitazione meridionale altrove diffusa nell'area del Parco e anche nei limitrofi Parco Nazionale dei Monti Sibillini a Nord e Parco Nazionale della Majella a Sud (Bartolozzi \& Maggini, 2005; Biscaccianti, dati inediti). Sebbene solo L. cervus sia specie tutelata dalla Direttiva Habitat, sarebbe opportuno considerare alla stessa stregua anche il congenere tetraodon, a tutti gli effetti equiparabile sotto il profilo biologico, ecologico e conservazionistico, ma ancor più significativo sotto il profilo biogeografico.

Cerambyx cerdo, pur non essendo propriamente una specie saproxilica (vedi sopra), viene di norma considerata tale perché la pluriennale attività trofica delle larve contribuisce a incrementare la disponibilità di legno morto su vecchi alberi vegeti, favorendo quindi la successione di importanti cenosi di invertebrati saproxilici (Buse et al., 2008a, 2008b). Nell'area in esame la specie è probabilmente più diffusa di quanto non appaia; tuttavia, mancando studi di dettaglio sulla componente saproxilica dei boschi di media e bassa quota del Parco, i dati relativi alla sua geonemia e alla composizione delle entomocenosi associate al suo habitat sono ancora notevolmente lacunosi.

Infine Osmoderma eremita, presente nell'area dal piano basale a quello montano, è una specie particolarmente importante perché rappresentativa della peculiare fauna associata alle cavità degli alberi, habitat nettamente in declino in Europa (Ranius et al., 2005). E' significativo il fatto che la specie sia stata ritrovata dopo vent'anni sempre nella Valle del Venacquaro, dove infatti permangono interessanti nuclei relitti di foresta vetusta. Come si è detto, nello stesso biotopo è stata riscontrata la presenza anche di Protaetia marmorata (Cetoniidae), specie rara che occupa lo stesso habitat di $O$. eremita. Nelle cavità arboree di maggiori dimensioni si sviluppano anche altri elementi stenotopi come ad esempio Prionychus ater (Fabricius, 1775) (Alleculidae), mentre quelle che si aprono alla base dei vecchi alberi ospitano specie differenti, tra cui Prionus coriarius (Linnaeus, 1758)
(Cerambycidae), Mycetochara linearis (Illiger, 1794), Pseudocistela ceramboides (Linnaeus, 1760) (Alleculidae) e altre.

Occorre osservare che nessuna delle specie citate in questo lavoro è stata inserita nei formulari standard dei siti Natura 2000 presenti all'interno del territorio del Parco, con l'unica eccezione di O. eremita, inserita in quelli della ZPS "IT7110128 - Parco Nazionale del Gran Sasso e Monti della Laga" e del SIC "IT7110209 - Primo tratto del Fiume Tirino e Macchiozze di S. Vito". Quest'ultima segnalazione, tuttavia, non sembra essere suffragata da alcun reperto, edito o inedito, ma deriva quasi certamente da una valutazione di presenza potenziale, dovuta al fatto che la specie è nota di una stazione molto prossima e con caratteristiche ambientali analoghe (Marotta et al., 1997).

Dai risultati riportati in questo contributo appare evidente che il territorio del Parco Nazionale del Gran Sasso e Monti della Laga può rivestire un ruolo particolarmente importante nella conservazione della fauna saproxilica e, più in generale, degli ambienti forestali in Italia, ma è necessario che vengano promossi e sostenuti ulteriori studi specifici su detta componente faunistica. Da un lato, infatti, la presenza accertata di ben cinque specie di coleotteri saproxilici di Direttiva Habitat è di per se un dato particolarmente significativo e difficilmente riscontrabile altrove in Europa; dall'altro va sottolineato che il ruolo di "specie ombrello" che queste potrebbero assumere è senza dubbio un elemento di grande rilievo per la tutela della biodiversità degli habitat cui sono associate.

\section{RINGRAZIAMENTI}

Ci è gradito ringraziare l'Ente Parco Nazionale del Gran Sasso e Monti della Laga per le autorizzazioni concesse e per il supporto logistico durante le ricerche sul campo, e Piermaria Corona (Università della Tuscia, Viterbo), responsabile del progetto di ricerca. Un particolare ringraziamento è dovuto anche a Luca Bartolozzi (Università "La Specola", Firenze), Giorgio Lalli (Università de L'Aquila), Maurizio Ettorre (Giulianova, TE), Carlo Artese (Ente Parco Nazionale del Gran Sasso e Monti della Laga, Assergi, $\mathrm{AQ}$ ), Emanuele Piattella (Università "La Sapienza", Roma) e Andrea Liberto (Roma), che a vario titolo hanno contribuito alla stesura di questo contributo. Un sentito ringraziamento infine a Pier Mauro Giachino (Regione Piemonte, Torino) per la rilettura critica del manoscritto. 
BIBLIOGRAFIA

Albert J., Platek M., Cizek L., 2012 - Vertical stratification and microhabitat selection by the Great Capricorn Beetle (Cerambyx cerdo) (Coleoptera: Cerambycidae) in open-grown, veteran oaks. European Journal of Entomology, 109(4): 553-559.

Audisio P., Ballerio A., Carpaneto G.M., Antonini G., Mancini E., Coletti G., Piattella E., De Biase A., 2003 - Osmoderma eremita s.l. in Europa meridionale: stato delle conoscenze e problemi di conservazione (Coleoptera, Cetoniidae). In: MAson F., NARDi G., Tisato M. (eds), Proceedings of the International Symposium "Dead wood: a key to biodiversity", Mantova, May 29-31, 2003. Sherwood, 95 (suppl. 2): 57-60.

Audisio P., Biscaccianti A.B., Carpaneto G.M., Chiari S., De Biase A., Maurizi E., Pace G., 2008 - Odontosphindus grandis, genere e specie nuovi per la fauna italiana (Coleoptera, Sphindidae). Bollettino della Associazione romana di entomologia, 63(1-4): 43-46.

Audisio P., Brustel H., Carpaneto G.M., Coletti G., Mancini E., Piattella E., Trizzino M., Dutto M., Antonini G., De BIASE A., 2007 - Updating the taxonomy and distribution of the European Osmoderma, and strategies for their conservation (Coleoptera: Scarabaeidae, Cetoniinae). Fragmenta entomologica, 39(2): 273-290.

Audisio P., Brustel H., Carpaneto G.M., Coletti G., Mancini E., Trizzino M., Antonini G., De Biase A., 2009 - Data on molecular taxonomy and genetic diversification of the European Hermit beetles, a species-complex of endangered insects (Coleoptera: Scarabaeidae, Cetoniinae, Osmoderma). Journal of Zoological Systematics and Evolutionary Research, 47(1): 88-95.

Bartolozzi L., Maggini L., 2005 - Insecta Coleoptera Lucanidae. In: Ruffo S., Stoch F. (eds), Checklist e distribuzione della fauna italiana. 10.000 specie terrestri e delle acque interne. Memorie del Museo Civico di Storia naturale di Verona, (s. II), sezione Scienze della Vita, 16: 191-192, con dati su CD-ROM.

Bartolozzi L., Sprecher-Uebersax E., 2006 - Lucanidae. In: Löbl I., Smetana A. (eds), Catalogue of Palaearctic Coleoptera. 3. Scarabaeoidea, Scirtoidea, Dascilloidea, Buprestoidea, Byrrhoidea. Apollo Books, Stenstrup: 63-77.

BELl R.T., 1998 - Where do the Rhysodini (Coleoptera) belong? Proceedings of the XX International Congress of Entomology, Firenze, August 25-31, 1996: 261-272.

BENSE U., 1995 - Longhorn Beetles. Illustrated key to the Cerambycidae and Vesperidae of Europe. Bockkäfer. Illustrierter Schlüssel zu den Cerambyciden und Vesperiden Europas. Margraf Verlag, Weikersheim, 512 pp.

BisCACCIANTi A.B., 2004 - Note su alcuni Longicorni dell'Appennino umbro-marchigiano (Italia centrale) (Coleoptera, Cerambycidae). Bollettino della Associazione romana di entomologia, 59(1-4): 43-88.

BisCACCIANTI A.B., 2006 - Gli studi sui longicorni (Coleoptera, Cerambycidae) della Majella e del Morrone. V Congresso Nazionale Federparchi, Catania-Trecastagni-Nicolosi, 1-4 giugno 2006, poster.

Biscaccianti A.B., Casalini R., 2007 - Considerazioni sul significato ecologico e biogeografico di alcuni Longicorni dei Monti Prenestini (Antiappennino laziale) (Coleoptera Cerambycidae). Bollettino della Società entomologica italiana, 139(1): 27-42.

Blasi C., Marchetti M., Chiavetta U., Aleffi M., Audisio P., Azzella M.M., Brunialti G., Capotorti G., Del Vico E., Lattanzi E., Persiani A.M., Ravera S., Tilia A., Burrascano S., 2010 - Multi-taxon and forest structure sampling for identification of indicators and monitoring of old-growth forest. Plant Biosystems, 144(1):160-170.

Bosso L., Rebelo H., Garonna A.P., Russo D., 2013 - Modelling geographic distribution and detecting conservation gaps in Italy for the threatened beetle Rosalia alpina. Journal for Nature Conservation, 21(2): 72-80.

Brustel H., 2004 - Contribution à la connaissance d'Osmoderma spp. dans le sud de l'Europe (Coleoptera, Cetoniidae). Le Coléoptériste, 7(3): 203-205.

BRustel H., GouIX N., 2011 - Coléoptères Rhysodidae en France: données complémentaires pour Rhysodes sulcatus (F., 1787) et incitation à la recherché d'Omoglymmius (s.s.) germari (Ganglbauer, 1892). L'Entomologiste, 67(6): 321-325.

Burakowski B., 1975 - Description of larva and pupa of Rhysodes sulcatus (F.) (Coleoptera, Rhysodidae) and notes on the bionomics of this species. Annales Zoologici, 32(12): 271-287.

Buse J., Ranius T., Assmann T., 2008a - An endagered longhorn beetle associated with old oaks and its possible role as an ecosystem engineer. Conservation Biology, 22(2): 329-337.

Buse J., ZABRAnSKy P., Assmann T., 2008b - The xylobiontic beetle fauna of old oaks colonised by the endangered longhorn beetle Cerambyx cerdo Linnaeus, 1758 (Coleoptera: Cerambycidae). Mitteilungen der Deutschen Gesellschaft für Allgemeine und Angewandte Entomologie, 16(2007): 109-112.

Calamini G., Maltoni A., Travaglini D., Iovino F., Nicolaci A., Menguzzato G., Corona P., Ferrari B., Di Santo D., ChiRICI G., LOMBARDI F., 2011 - Stand structure attributes in potential old-growth forests in the Apennines, Italy. L'Italia Forestale e Montana, 66(5): 365-381. 
Carpaneto G.M., Piattella E., Valerio L., 2005 - Insecta Coleoptera Scarabaeoidea. In: Ruffo S., Stoch F. (eds), Checklist e distribuzione della fauna italiana. 10.000 specie terrestri e delle acque interne. Memorie del Museo Civico di Storia naturale di Verona, (s. II), sezione Scienze della Vita, 16: 193-197, con dati su CD-ROM.

Castro A., Martínez de Murguía L., Fernández J., Casis A., Molino-Olmedo F., 2012 - Size and quality of wood used by Rosalia alpina (Linnaeus, 1758) (Coleoptera: Cerambycidae) in beech woodlands of Gipuzkoa (northern Spain). Munibe, 60: 77-100

Cecchi B., Bartolozzi L., 1997 - I coleotteri xilofagi e subcorticicoli del Parco Nazionale delle Foreste Casentinesi, Monte Falterona e Campigna. Bollettino della Società entomologica italiana, 129(2): 119-139.

Collalti D., D’Alessandro L., Marchetti M., Sebastiani A. (eds), 2009 - La carta tipologico-forestale della Regione Abruzzo. Volume generale. Regione Abruzzo, L'Aquila, 340 pp.

Consiglio CE, 1992 - Direttiva 92/43/CEE del Consiglio del 21 maggio 1992 relativa alla conservazione degli habitat naturali e seminaturali e della flora e della fauna selvatiche. Gazzetta Ufficiale delle Comunità Europee, L 206: 7-50.

Consiglio UE, 2006 - Direttiva 2006/105/CE del Consiglio del 20 novembre 2006 che adegua le direttive 73/239/CEE, 74/557/CEE e 2002/83/CE in materia di ambiente, a motivo dell'adesione della Bulgaria e della Romania. Gazzetta Ufficiale dell'Unione Europea, L 363: 368-408.

CONTARINI E., 2003 - La rarefazione della coleotterofauna xilofaga in rapporto alla gestione dei boschi. Atti del Convegno "Dagli alberi morti... la vita della foresta. La conservazione della biodiversità forestale legata al legno morto". Corniolo, 10 Maggio 2002. Parco Nazionale delle Foreste Casentinesi, Monte Falterona e Campigna: 40-43.

Conti F., AbBate G., Alessandrini A., Blasi C. (eds), 2005 - An annotated checklist of the Italian vascular flora. Palombi \& Partner, Roma, $420 \mathrm{pp}$

Costa A., 1854 - [Coleotteri. Parte $\mathrm{II}_{\mathrm{a}}$.] Coleotteri tetrameri. Sezione de’ Longicorni. In: Costa O.G. (ed.), Fauna del Regno di Napoli ossia enumerazione di tutti gli animali che abitano le diverse regioni di questo Regno e le acque che le bagnano contenente la descrizione de' nuovi o poco esattamente conosciuti con figure ricavate da originali viventi e dipinte al naturale. Stamperia Gaetano Sautto, Napoli [fogli 10-12]: 1-24.

COUNCIL OF Europe, 1979 - Convention on the conservation of European wildlife and natural habitats. European Treaty Series, 104: 1-12 + Annexes I-IV.

Cox K., Thomaes A., Antonini G., Zilioli M., De Gelas K., Harvey D., Solano E., Audisio P., McKeown N., Shaw P., MiNetTi R., BARtolozzi L., Mergeay J., 2013 - Testing the performance of a fragment of the COI gene to identify western Palaearctic stag beetle species (Coleoptera, Lucanidae). In: Nagy Z.T., Backeljau T., De Meyer M., Jordaens K. (eds), DNA barcoding: a practical tool for fundamental and applied biodiversity research. Zookeys, 365: 105-126.

DAJOZ R., 2000 - Insects and forests. The role and diversity of Insects in the forest environment. Lavoisier Publishing, Paris, 668 pp.

Demelt C., 1966 - II. Bockkäfer oder Cerambycidae. I. Biologie mitteleuropäischer Bockkäfer (Col. Cerambycidae) unter besonderer Berücksichtigung der Larven. In: DAHL F., DAHL M., PEus F. (eds), Die Tierwelt Deutschlands und der angrenzenden Meeresteile nach ihrer Merkmalen und nach ihrer Labensweise, 52. V.G. Fischer Verlag, Jena: 1-115, tafeln I-IX.

Di Santo D., Catonica C., Ciani L., Di Paolo S., Ferrari B., Giannini R., Paffetti D., Vettori C., 2010 - Il bosco vetusto di Fonte Novello. Biodiversità Italiana, 3: 28-31.

FABBRI R., 2010 - Segnalazioni faunistiche n. 99 - Lucanus tetraodon tetraodon Thunberg, 1806 (Insecta Coleoptera Lucanidae). Quaderni di Studi e Notizie di Storia Naturale della Romagna, 29(2009): 235-236.

Franciscolo M.E., 1997 - Fauna d'Italia, XXXV. Coleoptera Lucanidae. Calderini, Bologna, XI + 228 pp.

FrANKLIN J.F., SPIES T.A., 1991 - Composition, function, and structure of old-growth Douglas-fir forests. In: RUGGIERO L.F., Aubry K.B., CAREY A.B., HufF M.H. (eds), Wildlife and vegetation of unmanaged Douglas-fir forests. USDA Forest Service General Technical Report PNW-GTR-285, Portland (OR, USA): 91-110.

GILG O., 2004 - Forêts à caractére naturel: caractéristiques, conservation et suivi. Cahiers Techniques de l'ATEN, 74. ATEN, Montpellier, 96 pp.

GoвBI G., 1994 - Interessanti reperti di Cerambycidae italiani, specialmente delle regioni centro-meridionali (Coleoptera). Bollettino della Associazione romana di entomologia, 48(1-4) (1993): 83-101.

GrEDLER V.M., 1866 - Die Käfer von Tirol nach ihrer horizontalen und vertikalen Verbreitung. 2. Hälfte. Eberle Verlag, Bozen: 235-491.

Grove S.J., 2002 - Saproxylic insect ecology and the sustainable management of forests. Annual review of Ecology and Systematics, 33: 1-23.

Harvey D.J., Gange A.C., Hawes C.J., Rink M., 2011 - Bionomics and distribution of the stag beetle, Lucanus cervus (L.) across Europe. Insect Conservation and Diversity, 4(1): 23-38.

Horion A., 1961 - Faunistik der mitteleuropäischen Käfer, VIII. Clavicornia 2. A. Feyel, Überlingen-Bodensee, XV +375 pp.

HrusKa K., 1988 - I castagneti dei Monti della Laga (Italia Centrale). Braun Blanquetia, 2: 117-125. 
JONES C.G., LAWTON J.H., SHACHAK M., 1994 - Organisms as ecosystem engineers. Oikos, 69: 373-386.

Jurc M., Ogris N., Pavlin R., Borkovic D., 2008 - Forest as a habitat of saproxylic beetles on Natura 2000 sites in Slovenia. Revue d'Ecologie (La Terre et la Vie), 63: 53-66.

Keddy P.A., Drummond C.G., 1996 - Ecological properties for the evaluation, management, and restoration of temperate deciduous forest ecosystems. Ecological Applications, 6(3): 748-762.

Косн K., 1989 - Die Käfer Mitteleuropas. Ökologie. Band E3. Goecke \& Evers Verlag, Krefeld, 382 pp.

KRELl F.T., 1997 - Zur Taxonomie, Chorologie und Eidonomie einiger westpaläarktischer Lamellicornia (Coleoptera). Entomologische Nachrichten und Berichte, 40(4): 217-229.

LESNE P., 1901 - Synopsis des Bostrychides paléartiques. L'Abeille, 30: 73-136.

Löbl I., Smetana A. (eds), 2003 - Catalogue of Palaearctic Coleoptera. 1. Archostemata, Myxophaga, Adephaga. Apollo Books, Stenstrup, $819 \mathrm{pp}$.

Löbl I., Smetana A. (eds), 2004 - Catalogue of Palaearctic Coleoptera. 2. Hydrophiloidea, Histeroidea, Staphylinoidea. Apollo Books, Stenstrup, $942 \mathrm{pp}$.

LöBl I., SmetanA A. (eds), 2006 - Catalogue of Palaearctic Coleoptera. 3. Scarabaeoidea, Scirtoidea, Dascilloidea, Buprestoidea, Byrrhoidea. Apollo Books, Stenstrup, 690 pp.

Löbl I., Smetana A. (eds), 2007 - Catalogue of Palaearctic Coleoptera. 4. Elateroidea, Derodontoidea, Bostrichoidea, Lymexyloidea, Cleroidea, Cucujoidea. Apollo Books, Stenstrup, 935 pp.

LöBl I., Smetana A. (eds), 2008 - Catalogue of Palaearctic Coleoptera. 5. Tenebrionoidea. Apollo Books, Stenstrup, 670 pp.

Löbl I., Smetana A. (eds), 2010 - Catalogue of Palaearctic Coleoptera. 6. Chrysomeloidea. Apollo Books, Stenstrup, 924 pp.

Luigioni P., 1929 - I Coleotteri d'Italia. Catalogo sinonimico, topografico, bibliografico. Memorie della Pontificia Accademia delle Scienze, I Nuovi Lincei, (s. II), 13: 1-1160.

MAKARov K.V., 2008 - Larval chaetotaxy in the genus Rhysodes Dalman, 1823 and the position of Rhysodidae within Adephaga (Coleoptera). Proceedings of the XIII European Carabidologists Meeting, Blagoevgrad, August 20-24, 2007: 101-123.

Marotta O., Andreetti A., Osella G., 1997 - 19. Coleoptera Lucanoidea \& Scarabaeoidea (Insecta). In: Osella G., Biondi M., Di Marco C., Riti M. (eds). Ricerche sulla Valle Peligna (Italia centrale, Abruzzo). Quaderni di Provinciaoggi, L'Aquila, 23(1): 363-377.

Moritz C., 1994 - Defining evolutionarily significant units for conservation. Trends in Ecology and Evolution, 9: 373-375.

NÁDAi L., 2006 - A Bolboceratinae alcsalád magyarországi fajainak lelőhelyadatai (Coleoptera, Scarabaeoidea: Geotrupidae). Folia Historico Naturalia Musei Matraensis, 30: 205-210.

NARDi G., 2005 - Insecta Coleoptera Bostrichidae. In: RUFFo S., STOCH F. (eds), Checklist e distribuzione della fauna italiana. 10.000 specie terrestri e delle acque interne. Memorie del Museo Civico di Storia naturale di Verona, (s. II), sezione Scienze della Vita, 16: dati su CD-ROM.

Nieto A., AleXander K.N.A., 2010 - European Red List of saproxylic beetles. Publications Office of the European Union, Luxenbourg, VIII + 46 pp.

Oleksa A., Chybicki I.J., GAWronski R., Svensson G.P., BurczyK J., 2013 - Isolation by distance in saproxylic beetles may increase with niche specialization. Journal of Insect Conservation, 17(2): 219-233.

PAILl W., 2008 - Wiederfund von Bolbelasmus unicornis (Schrank, 1789) in den Wiener Donauauen (Coleoptera: Scarabaeoidea: Geotrupidae). Beitrage zur Entomofaunistik, 8(2007): 165-171.

PAPINI G., 1962 - Reperti. Rhysodes sulcatus Fabr. (Col. Rhysodidae). Bollettino della Associazione romana di entomologia, 17(2-3): 9.

Peez A., Kahlen M., 1977 - Die Käfer von Südtirol. Faunistiches Verzeichnis der aus der Provinz Bozen bisher bakannt gewoerdenen Koleopteren. Veroffentlichungen des Museum Ferdinandeum, Innsbruck, 57(2): 1-525.

PorTA A., 1923 - Fauna Coleopterorum Italica. I. Adephaga. Cicindelidae, Carabidae, Haliplidae, Hygrobiidae, Dyticidae, Gyrinidae, Rhisodidae, Paussidae. Stabilimento Tipografico Piacentino, Piacenza, VII + 285 pp.

PRUnier D., 1999 - Quelques observations sur la biologie d'Osmoderma eremita Scop. Le Coléoptériste, 35: 23-24.

RANius T., 2000 - Minimum viable metapopulation size of a beetle, Osmoderma eremita, living in tree hollows. Animal Conservation, 3 (1): 37-43.

RANIUS T., 2001 - Constancy and asynchrony of populations of a beetle, Osmoderma eremita living in tree hollows. Oecologia, 126 (2): 208-215.

RANIUS T., 2002a - Influence of stand size and quality of tree hollows on saproxylic beetles in Sweden. Biological Conservation, 103(1): 85-91.

RANius T., 2002b - Population ecology and conservation of beetles and pseudoscorpions living in hollow oaks in Sweden. Animal Biodiversity and Conservation, 25(1): 53-68.

Ranius T., Hedin J., 2001 - The dispersal rate of a beetle, Osmoderma eremita, living in tree hollows. Oecologia, 126(3): 363-370. 
Ranius T., NiLsson S.G., 1997 - Habitat of Osmoderma eremita Scop. (Coleoptera: Scarabaeidae), a beetle living in hollow trees. Journal of Insect Conservation, 1(4): 193-204.

Ranius T., Aguado L.O., Antonsson K., Audisio P., Ballerio A., Carpaneto G.M., Chobot K., Gjurašin B., Hanssen O., Huijbregts H., Lakatos F., Martin O., Neculiseanu Z.Z., Nikitsky N.B., Paill W., Pirnat A., Rizun V.V., Ruicănescu A., Stegner J., SÜda I., SzwaŁko P., Tamutis V., Telnov D., Tsinkevich V., Versteirt V., Vignon V., Vögeli M., ZACH P., 2005 - Osmoderma eremita (Coleoptera, Scarabaeidae, Cetoniinae) in Europe. Animal Biodiversity and Conservation, 28(1): 1-44.

Reitter E., 1894 - Übersicht der Arten der Coleopteren Gattung Morimus Serv. Wiener Entomologische Zeitung, 13(2): $43-44$.

RufFo S., STосн F. (eds), 2005 - Checklist e distribuzione della fauna italiana. 10.000 specie terrestri e delle acque interne. Memorie del Museo Civico di Storia naturale di Verona, (s. II), sezione Scienze della Vita, 16: 1-307, con dati su CD-ROM.

Russo D., Cistrone L., Garonna A.P., 2011 - Habitat selection by the highly endangered long-horned beetle Rosalia alpina in Southern Europe: a multiple spatial scale assessment. Journal of Insect Conservation, 15(5): 685-693.

SAma G., 1988 - Fauna d'Italia, XXVI. Coleoptera Cerambycidae. Catalogo topografico e sinonimico. Calderini, Bologna, XXXVI + $216 \mathrm{pp}$.

SAMA G., 2002 - Atlas of the Cerambycidae of Europe and Mediterranean Area. 1: Northern, Western, Central and Eastern Europe. British Isles and Continental Europe from France (excl. Corsica) to Scandinavia and Urals. Kabourek, Zlin, 173 pp.

SAma G., 2005 - Insecta Coleoptera Cerambycidae. In: Ruffo S., Sтосн F. (eds), Checklist e distribuzione della fauna italiana. 10.000 specie terrestri e delle acque interne. Memorie del Museo Civico di Storia naturale di Verona, (s. II), sezione Scienze della Vita, 16: 219-222, con dati su CD-ROM.

SPARACIO I., 1994 - Osmoderma cristinae n. sp. di Sicilia (Insecta Coleoptera: Cetoniidae). Il Naturalista sicliano, (s. IV), 17(3-4): 305-310.

SpARACIO I., 2000 - Osservazioni sulle Osmoderma Le Peletier et Audinet-Serville europee con descrizione di una nuova specie dell'Italia meridionale (Coleoptera Cetoniidae). Il Naturalista sicliano, (s. IV), 24 (3-4): 225-239.

SPEIGHT M.C.D., 1989 - Saproxylic invertebrates and their conservation. Nature and Environment series, 42. Council of Europe, Strasbourg, $66 \mathrm{pp}$.

TAmmaro F., 1998 - Diversità floristica e vegetazionale sul Gran Sasso e sui Monti della Laga. In: BurRi E. (ed.), Aree protette in Abruzzo. Contributi alla conoscenza naturalistica e ambientale. Carsa Edizioni, Pescara: 35-58.

TAUZIN P., 1994 - Le genre Osmoderma Le Peletier et Audinet-Serville 1828 (Coleopt., Cetoniidae, Trichiinae, Osmodermatini). Systématique, biologie et distribution (Deuxième partie). L'Entomologiste, 50(4): 217-242.

Tondi G., 1996 - 37. La betulla della Laga. In: Dinelli A., GuARrera P.M. (eds), Censimento del patrimonio vegetale del Lazio, Quaderno n. 2. Ambienti di particolare interesse naturalistico del Lazio. Tipar Poligrafica Editrice, Roma: 170-173.

TRAVÉ J., 2003 - Dead wood and saproxylic complex in the Massane forest. Role in the conservation of Invertebrates. In: BowEN C.P. (ed.), Proceedings of the second pan-European conference on Saproxylic Beetles. People's Trust for Endangered Species, London: 68-71.

Zilioli M., PitTino R., 2004 - Un reperto eccezionale: Lucanus tetraodon Thunberg in Lombardia. Atti della Società italiana di Scienze naturali e del Museo civico di Storia naturale di Milano, 145(2): 301-306. 